\title{
Macromere cell fates during sea urchin development
}

\author{
R. ANDREW CAMERON*, SCOTT E. FRASER, ROY J. BRITTEN and ERIC H. DAVIDSON \\ Division of Biology, California Institute of Technology, Pasadena, California 91125, USA
}

* Author for correspondence

\begin{abstract}
Summary
This paper examines the cell lineage relationships and cell fates in embryos of the sea urchin Strongylocentrotus purpuratus leading to the various cell types derived from the definitive vegetal plate territory or the $v g_{2}$ tier of cells. These cell types are gut, pigment cells, basal cells and coelomic pouches. They are cell types that constitute embryonic structures through cellular migration or rearrangement unlike the relatively non-motile ectoderm cell types. For this analysis, we use previous knowledge of lineage to assign macromeres to one of four types: VOM, the oral macromere; VAM, the aboral macromere, right and left VLM, the lateral macromeres. Each of the four macromeres contributes progeny to all of the cell types that descend from the definitive vegetal plate. Thus in the gut each macromere contributes to the
\end{abstract}

esophagus, stomach and intestine, and the stripe of labeled cells descendant from a macromere reflects the re-arrangement of cells that occurs during archenteron elongation. Pigment cell contributions exhibit no consistent pattern among the four macromeres, and are haphazardly distributed throughout the ectoderm. Gut and pigment cell contributions are thus radially symmetrical. In contrast, the VOM blastomere contributes to both of the coelomic pouches while the other three macromeres contribute to only one or the other pouch. The total of the macromere contribution amounts to $60 \%$ of the cells constituting the coelomic pouches.

Key words: cell lineage, embryo, vegetal plate, Strongylocentrotus purpuratus, macromere.

\section{Introduction}

The vegetal plate is one of five tissue territories that are identified by unique patterns of gene expression, an individual cell lineage history and one or more cell types in regularly developing sea urchin embryos (Davidson, 1989; Cameron and Davidson, 1991). Lineage tracing studies carried out on Strongylocentrotus purpuratus embryos demonstrate that each of these territories derives from a specific set of founder cells that segregate during cleavage. These founder cells and their immediate descendants are the basic units in which localized regulatory information, or intercellular interactions, may lead to patterns of spatially restricted gene expression (Davidson, 1989). Some of these territories are relatively simple, for example, the aboral ectoderm or the skeletogenic mesenchyme, in that they generate only one or two cell types. At the other extreme, the definitive vegetal plate which is considered in this communication is the most complex in fate. This territory contributes many different cell types including gut and the four cell types of the secondary mesenchyme, viz. basal cells, coelomic pouches, pigment cells and circumesophageal muscles (Cameron and Davidson, 1991). Pigment cells are large vesiculated cells filled with echinochrome pigment and intercalated in the ectoderm (Young, 1958; Gibson and Burke, 1985,
1987). Basal cells are branched, flattened cells which come to lie beneath the ectoderm in the pluteus stage (Ryberg and Lundgren, 1977; Burke, 1978). The coelomic pouches are a group of cells which separate from the tip of the archenteron when the mouth forms (Gustafson and Wolpert, 1963a), and the circumesophageal muscles arise from some of the coelomic pouch cells (Burke and Alvarez, 1988). All of these cell types differentiate relatively late, compared to those deriving from aboral ectoderm or skeletogenic mesenchyme (Davidson, 1986, 1989), and at late cleavage the vegetal plate territory consists of a set of possibly pluripotent blastomeres that are at present identified only by past and future lineage, and by their spatial position in the canonical cleavage pattern of the embryo. Since, in the undisturbed embryo, the founder cells of the vegetal plate territory give rise exclusively to the gut and secondary mesenchyme, their molecular state must also be different from those of other territories from early on. As an initial exploratory step in identifying the molecular features that might distinguish the cells of the vegetal plate territory, we have carried out a detailed analysis of the cell fates displayed by the clones that arise from the vegetal plate.

The definitive vegetal plate or $v^{2} g_{2}$ tissue territory derives from the 8-cell blastomeres of the vegetal half. As shown in Fig. 1, these cells divide unequally to form 


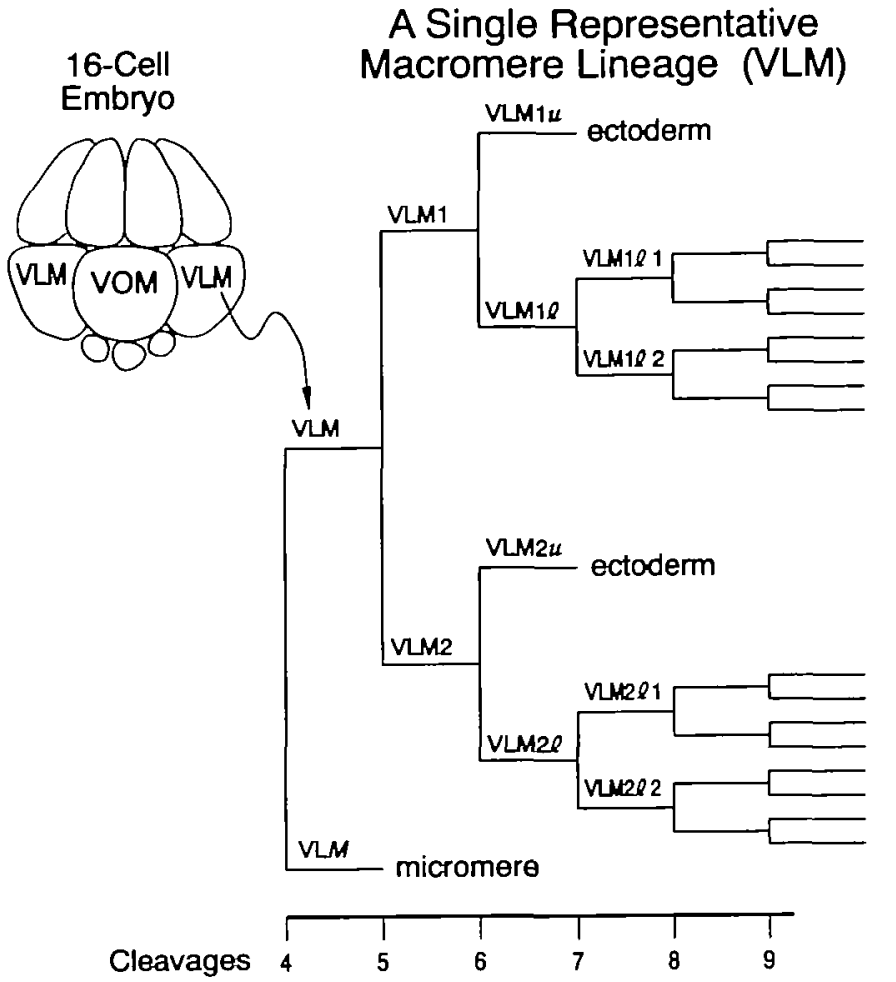

micromeres and macromeres. The micromeres divide unequally to form large micromeres, which are the founders of the primary skeletogenic mesenchyme cells that ingress into the blastocoel, and small micromeres (VAMs, VOMs, right and left VLMs), which remain at the tip of the advancing archenteron during gastrulation and contribute to the coelomic pouches (Pehrson and Cohen, 1986; Tanaka and Dan, 1990). The macromeres divide radially at the fifth division, then horizontally at the sixth division to form two tiers of cells. The lower tier contributes to the vegetal plate, part of which invaginates to form the gut at gastrulation. The upper, more equatorial tier comprises the VAM1u, VAM2u, VOM1 $u$, VOM $2 u$, right and left VLM1 $u$, right and left VLM $2 u$ cells and contributes to both the oral and aboral ectoderm (Fig. 1). Thus, the founder cells for the definitive vegetal plate are the most vegetal tier (VAM1 $l$, VAM2l, right and left VLM1l, right and left VLM2 $l$, VOM1 $l$ and VOM2l) (Cameron et al. 1987; Cameron and Davidson, 1991). Furthermore, the sister cell of each vegetal plate founder cell derived from a macromere is the founder for a unique ectodermal tract (Fig. 1).

How vegetal plate territory blastomeres segregate the diverse cell types formed from it is not known. It is possible that all four macromeres contribute to all cell types equally, i.e. the contribution is radially symmetrical; in contrast, one or another cell type may derive from a single macromere, i.e. a radial asymmetry in cell fate exists. The cell types derived from the vegetal plate territory reach their final position in the embryo as a result of morphogenetic movements such as gastrulation, evagination of the coelomic pouches, and migration of pigment and basal cells. The timing of

\section{Proposed \\ Fates}
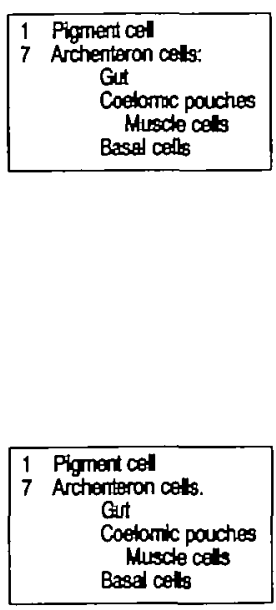

founder cell segregation for the various cell types must precede this migration. Each macromere contributes both ectoderm and vegetal plate derived cell types, and the ectodermal contributions are unique and recognizable (Cameron et al. 1987). Thus the injection of lineage tracer into a given macromere results in a unique ectodermal labeling pattern and permits the assignment of the vegetal plate cell labeling patterns to a particular macromere type (VOM, VAM, right or left VLM). We have utilized this method to determine the vegetal plate cell types produced by each of the four macromeres.

\section{Materials and methods}

\section{Embryo culture and preparation for injection}

Sea urchin embryos were prepared for iontophoretic injection using previously reported methods (Cameron et al. 1989, 1990). Briefly, Strongylocentrotus purpuratus gametes were obtained by electric shock or injection of $0.5 \mathrm{M} \mathrm{KCl}$ into the perivisceral coelom. Eggs were collected in filtered $(0.45$ micrometer pore size) natural sea water with $1 \mathrm{mM} 1,2,3$ aminotriazole (ATA) (Sigma, Inc.) and dejellied in pH 5.0 sea water. Semen was kept undiluted on ice. Eggs were electrostatically fixed to $60 \mathrm{~mm}$ Petri dish lids treated with $1 \%$ protamine sulfate (McMahon et al. 1985). The eggs were fertilized with a freshly diluted sperm suspension and the embryos allowed to develop to the sixteen-cell stage. Typically $10-12$ eggs were attached to a single dish and about 20 dishes were prepared in a single experiment. All sea waters used through the incubation to sixteen-cell stage contained $1 \mathrm{~mm}$ ATA.

Just before injection, the embryos were rinsed three times in calcium- and magnesium-free sea water to soften the tough extracellular coats and facilitate penetration with microelectrodes. Following injection, calcium and magnesium ion 
concentrations were returned to those of artificial sea water, the dish was then washed several times with 0.45 micrometer filtered sea water containing penicillin $\left(20\right.$ units $\left.\mathrm{ml}^{-1}\right)$ and streptomycin $\left(50 \mu \mathrm{g} \mathrm{ml}^{-1}\right)$ and the embryos were incubated in the dark for $72 \mathrm{~h}$ at $16^{\circ} \mathrm{C}$. The embryos have developed to the pluteus stage over this interval and consist of about 1800 cells.

\section{Fluorescent dye injection and analysis}

Single macromeres of sixteen-cell embryos were impaled with thin-walled aluminosilicate glass microelectrodes possessing tip resistances in the range of $10-50$ megohoms. The electrodes were filled at the tip with $50 \mathrm{mg} \mathrm{m}^{-1}$ lysinated rhodamine dextran (LRD) (Molecular Probes) and backfilled with $1.2 \mathrm{M} \mathrm{LiCl}$. Fluorescent dextan was ejected with positive current pulses $(200 \mathrm{~ms}, 5$ to $10 \mathrm{nA})$ at $2 \mathrm{~Hz}$ for 20 to $60 \mathrm{~s}$. Sometimes, injected embryos were fixed in $1-2 \%$ formaldehyde in filtered sea water for $30-90 \mathrm{~min}$. Microscopic views of wet mounted live or fixed plutei were recorded on an optical disc recorder through a light-intensifying camera (SIT) attached to an image processing system (Imaging Technologies). Selected samples were viewed on a Bio-Rad $600 \mathrm{C}$ laser scanning confocal microscope and optical series stored for later processing. Composites of fluorescent and bright-field images or stereo pairs were prepared from stored optical series and recorded on $35 \mathrm{~mm}$ Ektachrome film directly from the video monitor.

\section{Nuclear staining}

Aliquots of control embryos from injection experiments were fixed for $1 \mathrm{~h}$ in $1 \%$ formaldehyde in filtered sea water and then washed several times with filtered sea water. These fixed embryos were stained for about $10 \mathrm{~min}$ with $0.5 \mathrm{mg} \mathrm{m}^{-1}$ propidium iodide diluted from a stock solution with filtered sea water, then rinsed with filtered sea water and mounted for observation.

\section{Results}

In order to correlate the ectodermal contribution from macromeres with the vegetal plate contribution, optical sections of embryos labeled at the 16-cell stage were collected with the laser scanning confocal microscope (LSCM), processed into a single frame, and combined with a bright-field image. Composite images were compared to published 8-cell lineage tracer results of Cameron et al. (1987) in order to identify the macromere that had been injected in each case. Representative composite images for each of the three classes of patterns observed are presented in Fig. 2, with the bright-field component shown in green and the fluorescent component in yellow. Ectodermal labeling in the right anal plate, that is, aboral ectoderm immediately adjacent to the anus and posterior to the ciliated band, results from injection of a right VLM blastomere (Fig. 2A); and similarly, the left anal plate is labeled when the left VLM blastomere is injected. Labeling in the posterior apex of the aboral ectoderm where the body rods of the skeleton are closely apposed results from injection of a VAM blastomere (Fig. 2B). Labeling in the supra-anal ectoderm between the ciliated band and anus, and in oral ectoderm between the mouth and ciliated band, results from injection of LRD into the VOM blastomere (Fig. 2C). In addition, pigment cells, basal cells and gut sectors were also labeled in each specimen as discussed in detail below. In no case were the skeletogenic (or primary) mesenchyme cells labeled, indicating that the progenitors of these cells had completely segregated from their macromere sister-cells before the injections were performed. Since the four macromeres of the 16-cell stage are morphologically indistinguishable from one another, random injection of macromeres should result in a distribution of 1:2:1 between the VOM, VLM (right and left) and VAM blastomeres, respectively. The 44 successfully injected embryos involved in this study were scored as 10 VOM, 26 VLM and 8 VAM injections. By Chi-square analysis, this distribution is not different from the expected distribution, 11:22:11 (Snedecor and Cochran, 1979; $\chi^{2}=1.6363$ with 2 degrees of freedom).

The labeling patterns observed confirm that each macromere contributes progeny to all of the vegetal plate derivatives including the stomach, intestine and esophagus regions of the gut and the various secondary mesenchyme cell types (Cameron and Davidson, 1991). The tract of cells contributed to the gut by each type of macromere roughly coincides with the position of that blastomere in the spherical blastula before the postgastrular distortion of the ectoderm. Thus, for example, the VAM blastomere gut contribution forms an irregular stripe along the aboral aspect of the gut tube while the VOM blastomere contribution lies nearest to the oral ectoderm on the concave side of the curved gut tube. This can be seen in Fig. 2A-C.

The pigment cells tagged by each macromere injection were distributed haphazardly throughout the ectoderm, both within and outside the ectodermal tract contributed by their sister cells, VAM1 $u$, VAM $2 u$, VOM1 $u$, VOM $2 u$, right and left VLM1 $u$, right and left VLM2 $u$ as shown in Fig. 2A-C. Pigment cells, both labeled and unlabeled, appear more numerous in the tips of the forming arms and the posterior apex. Several labeled cells are often closely apposed in these areas. This may be due to the higher cell densities at the apex and arm tips as compared to the thin epithelial sheets of which the other ectodermal regions are composed. No reproducible arrangement of labeled pigment cells was observed for any of the macromeres. One to a few labeled non-pigmented cells with morphology identical to that described for basal cells were also observed in various positions in the blastocoel.

Labeled progeny of macromere injections were also evident in the clumps of cells alongside the esophagus, the coelomic pouches. At. this stage, the coelomic pouches consist of closely packed cells, and there is little or no lumen. In Fig. 3A is shown a LSCM composite image assembled as described above, and focused on the coelomic pouch cells in a pluteus stage embryo derived from an injected VAM macromere. Portions of gut are seen to be labeled, and two areas of fluorescence are evident in one coelomic pouch. In Fig. 3B, a similarly labeled pluteus reveals only a single 
sphere of label in the pouch. We cannot exclude the possibility that each labeled region consists of two labeled cells, although the circular appearance and diameter of the labeled areas suggest one cell per labeled sphere. We found that the VAM, right VLM and left VLM blastomeres each invariably contributed to only one pouch. In contrast, when the VOM macromere was injected with fluorescent lineage tracer, labeled cells appear in both of the coelomic pouches. In Fig. 4A,B, composite images of one embryo at two different focal planes are shown. Each composite was assembled from images of one optical section illuminated alternately with transmitted light and epifluorescent illumination. In Fig. 4A, the focus is set through the middle of the embryo so that the oral ectoderm, coelomic pouches and posterior end of the body rods are in focus. Lineage tracer is evident in what appear to be four to six cells in each of the coelomic pouches. Scattered labeled pigment cells at the posterior tip are also in focus. In Fig. 4B, the focus is set at the anal plate ectoderm, where a number of pigment cells appear as dark spots in the ectoderm. The ciliated band, the edge of the anus and scattered pigment cells are labeled with lineage tracer, definitively identifying the injected macromere as VOM.

In order to evaluate the relative contribution of macromere descendants to the coelomic pouches, the total number of cells in each pouch was estimated from fixed embryos labeled with propidium iodide. Typical propidium-iodide-stained embryos imaged as series of focal planes are depicted in Fig. 5A,B. Nuclei were counted in LSCM images prepared as projections or stereo pairs of through-focus series. Within one culture batch the mean number of cells in each pouch was about $10(n=5)$ with a range of $8-12$. In Table 1 are displayed the average number of coelomic pouch cells derived from each macromere, as estimated from lineage tracing. The VAM blastomere contributes one or two cells to either the left or right pouch. The lateral macromeres contribute an average of two cells to the coelomic pouch on the side of the embryo closest to its ectoderm contribution; so, for example, the left lateral macromere contributes to the left coelomic pouch. The oral macromere, VOM, contributes $4-6$ cells to both pouches. Pehrson and Cohen (1986) estimated that the small micromeres contribute four cells to each pouch in Strongylocentrotus purpuratus. Thus, of the approximately 10 cells in each coelomic pouch, the small micromeres contribute about $40 \%$, and the four macromeres the remainder.

Table 1. Coelomic pouch contributions

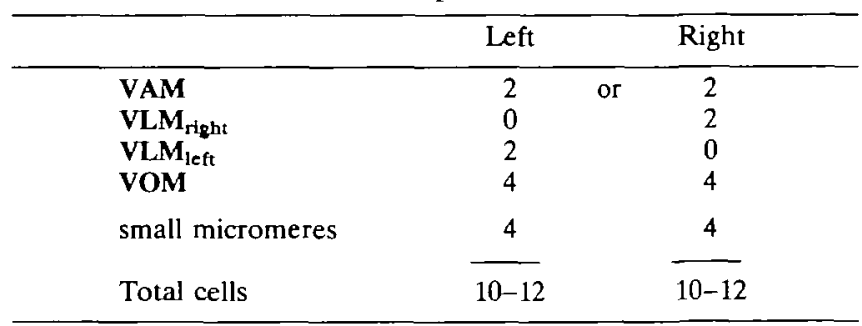

Fig. 2. Whole mounts of $72 \mathrm{~h}$ sea urchin embryos injected with lysyl rhodamine dextran lineage tracer in one macromere at the 16-cell stage. Each panel is a composite video image derived from two laser scanning confocal microscope image sets, a through-focus series with either epifluorescent or transmitted illumination. The fluorescent image is depicted in yellow and the transmitted light one in green. (A) An embryo injected in the right VLM blastomere and viewed from the right side. The anal plate aboral ectoderm, the lateral aboral aspect of the gut, and scattered pigment cells contain lineage tracer. (B) An embryo injected in the VAM blastomere and viewed from the anal side. The diagnostic features of this blastomere injection are labeling in the aboral ectoderm of the posterior apex and the aboral aspect of the gut. (C) A fixed embryo injected in the VOM blastomere and viewed from the anal side. The oral aspect of the gut, the ciliated band nearest to the anus and scattered pigment cells are labeled. Two regions on either side of the esophagus appear labeled. The skeletal rods of this embryo were dissolved during fixation.

Fig. 3. Views of $72 \mathrm{~h}$ embryos as described in Fig. 2 but confined to the regions of the coelomic pouches. $S$, stomach; E, esophagus. (A) An embryo injected in a VAM blastomere. It is viewed from the abanal side with the oral region toward the top. The coelomic pouches

(arrowheads), anterolateral rods, esophagus and the left edge of the mouth are in focus. In addition to the labeled cells in the ectoderm and gut, two spots of fluorescence are evident in the left coelomic pouch. (B) An embryo injected in the VAM blastomere and viewed from the anal side with the oral surface toward the left. The right coelomic pouch (arrowhead) contains one region of fluorescent marker. Fig. 4. Two whole-mount views of a $72 \mathrm{~h}$ sea urchin embryo injected in the VOM blastomere with the oral ectoderm toward the right. A single epifluorescently illuminated focal plane depicted in yellow is superimposed on a transmitted light image of the same plane depicted in green. (A) The image at the level of the coelomic pouches with the posterior apex also in focus. The fluorescent label is evident in groups of $4-6$ cells in each pouch. Labeled pigment cells can also be seen at the posterior tip. (B) The image at the level of the anal ectoderm. The ectodermembedded pigment cells are sharply in focus and fluorescent label is in the ciliated band nearest the anus and in a few pigment cells. Fluorescent signal from the out-of-focus posterior pigment cells is completely absent in this view,

Fig. 5. Views of uninjected $72 \mathrm{~h}$ embryos fixed and stained with propidium iodide nuclear stain. (A) A stereo image pair taken from a focus series that excludes the aboral ectoderm nearest the viewer and the anal ectoderm farthest from the viewer. The right coelomic pouch is clearly separated from the esophagus while the left one is partly obscured by it. (B) A through-focus series of images processed into one frame. The series lies in the region of the stomach and below the esophagus in this embryo. Two circles of coelomic pouch cells are evident between the stomach and oral ectoderm.

\section{Discussion}

This study shows that each macromere contributes to all of the cell types that derive from the definitive vegetal plate tissue territory. The position in the ectoderm of 

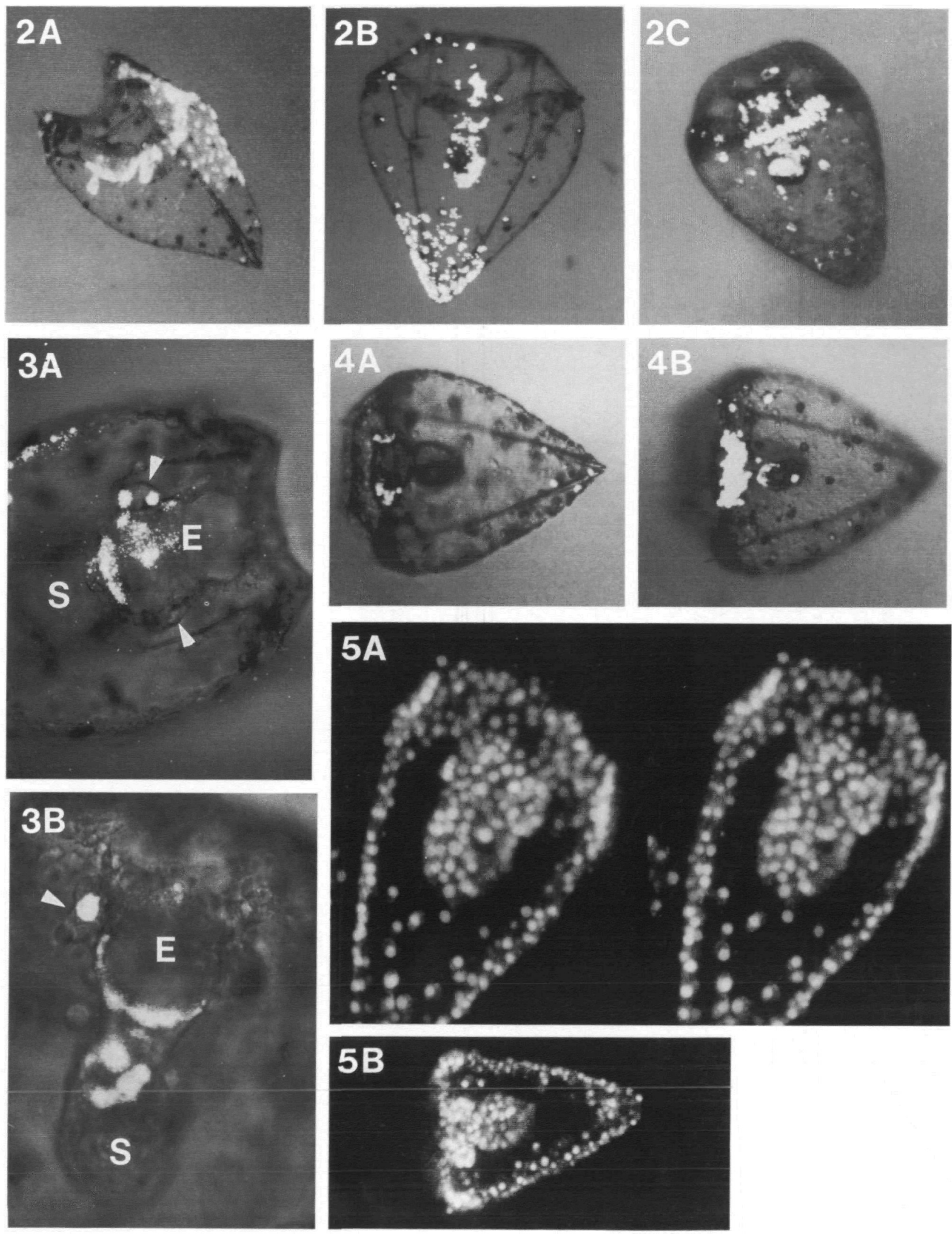

pigment cells and basal cells derived from each macromere are not consistent, from embryo to embryo, nor are they limited to any particular region. The contributions of each macromere to these migratory cell types are approximately equal in number and haphazardly arranged in the embryo. However, one radially asymmetrical contribution was observed. The VOM macromere contributes to both coelomic pouches while the VAM, left VLM and right VLM blastomeres each contribute to only one.

We now consider the possible founder cell origins for each of the structures that arise from the definitive vegetal plate and the cell types of which they are composed. All of these structures derive from cells that during development undergo more movement with respect to their neighbors than do the relatively nonmotile ectoderm cells. At the canonical 128-cell stage, in several species of sea urchins, the vegetal plate appears as concentric rings of cells encircling the four small micromeres (Morrill and Santos, 1985; Amemiya, 1989; Amemiya et al. 1982). The cells that are probably the small micromeres possess distinctly different surfaces. The circular arrays of cells preserve the furrows derived from each previous radial division. The innermost ring of eight cells consists of the large micromeres whose progeny are destined to ingress and become the skeletogenic mesenchyme cells (VAMk, VOMk, right and left VLMk; see Fig. 1). The next ring of 16 cells comprises the vegetal plate precursors, which have undergone the radial 7 th division, and are called $v e g_{2}$ in the terminology of Hörstadius (1939). Thus the next horizontal divisions, which will give rise to two rings of cells at the canonical 256-cell stage, would be the first in this sub-lineage at which differences in contribution to vegetal plate derivatives along the animal-vegetal axis could be observed. The outermost ring of 16 cells are the precursors of the ectoderm which derives from the vegetal half or the $v e g_{1}$ of Hörstadius (1939), i.e. the VAM1u, VAM2u, VOM1u, VOM2u, right and left VLM1 $u$, right and left VLM2 $u$ cells.

\section{(i) Pigment cells}

Pigment cells or echinophores are elongate, branched cells dispersed throughout the ectoderm which are filled with pigment granules. Gibson and Burke (1985) showed that the echinophores of Strongylocentrotus purpuratus are exclusively recognized by a monoclonal antibody to a cell surface protein, that is first detected in eight nonpigmented cells of the vegetal plate just after skeletogenic mesenchyme cell ingression. The position of these marked cells appears to be in the region of the $v^{2} g_{2}$ tier (Gibson and Burke, 1985). During the first third of archenteron invagination, antibody-positive cells are detected in the blastocoel, at the tip of the archenteron and in the vegetal plate. By the time the mouth has formed approximately 30 antibody-positive cells containing pigment granules are dispersed within the ectoderm. If all of the macromere sub-lineage cells that contribute to the vegetal plate territory continue to divide equally until the end of cleavage at the 9th division, which is probably the case, then there will be
16 cells derived from each macromere or 64 cells in the early blastula vegetal plate territory (Fig. 1). Since the pigment cell progenitors number about eight in the 400cell mesenchyme blastula (Gibson and Burke, 1985) and each macromere contributes an equal number, the echinophore founder cells must be segregated at the 8th or 9 th cleavage. These founder cells could arise as two sister cells which would be contiguous in the blastula wall or at the 9th division as independent segregants of each branch of the macromere sub-lineage (Fig. 1). Because when first observed the echinophore founder cells appear evenly distributed in the vegetal region (Gibson and Burke, 1985) and are therefore probably not contiguous, we propose that pigment cell founders segregate at the 9th division, one in each macromere sub-lineage (Fig. 1). Furthermore, independent developmental decisions for pigment cell differentiation must obtain if these cells are separated from one another in the vegetal plate.

\section{(ii) Basal cells}

A population of cells included in the secondary mesenchyme spans the blastocoel and connects the epidermis, the skeletal rods and muscles of the pluteus (Ryberg and Lundgren, 1977; Burke, 1978). Differential interference contrast microscopy and indirect immunocytochemistry suggest there are about 21 cells of this class in the pluteus stage (Tamboline and Burke, personal communication). Lineage tracer labeling reported here would predict up to about 16 basal cells total. The interval of founder cell segregation is unknown.

\section{(iii) Gut}

The archenteron invaginates in a two-step process which involves a cell shape change in the vegetal plate followed by re-arrangements of the cells in the archenteron wall that serve to extend the column across the blastocoel (Ettensohn, 1984, 1985; Hardin and Cheng, 1986). After gastrulation is completed with the formation of the mouth, the archenteron consists of about 100 cells. By the pluteus stage, the gut consists of 425 cells and three regions: esophagus, stomach, and intestine, separated by two muscular sphincters (Burke, 1980; Burke and Chia, 1980). The labeled tract of cells from each macromere forms an irregular band extending through all three gut regions. The irregularities reflect the cellular re-arrangements that occur with archenteron elongation (Cameron et al. 1987). The labeled tracts of cells do preserve the position of their precursor cells in the radially symmetrical gastrula; for example, the tract derived from the VAM lies in the aboral aspect of the gut while the VOM-derived gut tract is closest to the oral ectoderm. At the 6th cleavage, the vegetal plate arises as 8 cells, of which two each derive from a single macromere. By the 9th cleavage, the vegetal plate cells which remain after subtracting the eight cells that we proposed to be pigment cell founders would number 56 (Fig. 1). Thus only one further division would be required to arrive at the 100 cells of the complete archenteron after 
invagination commences (see Nislow and Morrill, 1988).

\section{(iv) Coelomic pouches}

The coelomic pouches consist of two groups of cells, which evaginate from the archenteron where it meets the ectoderm to form the mouth (Gustafson and Wolpert, 1963a,b). These structures proliferate after the onset of larval feeding and form adult structures, including the rudiment that becomes the oral surface of the adult sea urchin (Pearse and Cameron, 1991). At $72 \mathrm{~h}$ post-fertilization in Strongylocentrotus purpuratus, the coelomic pouches consist of about 10 cells each, four of which derive from the small micromeres. Burke and Alvarez (1988) report seven muscle cells and eight non-muscle cells in the coeloms of $84 \mathrm{~h}$ embryos reared under slightly different culture conditions. Macromeres were thought to contribute to the coelomic pouches based on their contribution to the archenteron from which the pouches derive (Czihak, 1971; Okazaki, 1975; Hörstadius, 1939). However, no direct demonstration of this contribution has been made until this work. The lineage tracing reported here demonstrates that about six of the ten cells in each pouch derive from macromeres, and that the oral macromere contributes to both pouches while the other three macromeres contribute to only one. The earliest division at which the macromere contribution could be segregated is near the end of cleavage, at the 7 th and 8 th division. Optical sections (data not shown) demonstrate that the center of the coelomic pouches usually lies somewhat below the midline of the esophagus. This is best illustrated in the clear delineation of the paired circles of cells in Fig. 5B. This position could result from an asymmetrical location of the evagination of the two sacs toward the oral side, and account thus for the contribution of the VOM cell to both sacs. This argument implies that the coelomic sac evagination point forms at the position on the archenteron wall where the boundary between the tract of cells derived from the VOM macromere and the tract from its neighboring lateral (VLM) macromeres.

That the coelomic pouches include all of the progeny of the small micromeres is demonstrated by staining for a histone H1 subtype (Pehrson and Cohen, 1986) and for 5-bromodeoxyuridine (BrdU) after pulse-chase labeling at an earlier stage (Tanaka and Dan, 1990). The small micromere founder cells arise at 5 th cleavage with the formation of VAMs, VOMs, right and left VLMs (Cameron and Davidson, 1991). It has been argued that the small micromeres may be the primordial germ cell lineage in sea urchins. The evidence supporting such a role involves their lack of continuing division and their position in the coelomic sacs at the end of larval life. From this position they might contribute the germ cells to the gonad, which forms from the coelomic lining early in post-metamorphic development (MacBride, 1903; Houk and Hinegardner, 1980, 1981). However, numerous cell divisions in fact occur in this structure during larval development and no large undivided cells have been figured in reviews of larval development. Furthermore, Tanaka and Dan (1991) mention that BrdU staining disappears later in development, implying that these small micromere derivatives do divide further.

After the coelomic pouches are established and begin to increase in cell number, about 14 cells extend processes around the esophagus to form the circumesophageal muscles (Burke and Alvarez, 1988). The pseudopods of the muscle-forming cells stain for muscle tropomyosin (Ishimoda-Takagi et al. 1984) and actin (Burke and Alvarez, 1988). The time at which the muscle cell population segregates is unknown. Only about one half of the cells of the coelomic pouch are muscle cells, and therefore it is possible that the dichotomy in origin of the coelomic pouch cells between macromeres and small micromeres is preserved.

The vegetal plate is the most complex tissue territory in the sea urchin embryo. It includes precursors to five different structures made up of one or more cell types. Yet this territory derives from only eight cells which segregate at the 64-cell stage: VAM1 $l$, VAM2 $l$, right and left VLM1 $l$, right and left VLM2 $l$, VOM1 $l$ and VOM $2 l$. The structures derived from the vegetal plate tissue territory differentiate later than those of other territories and only after a phase of relatively complex cellular movements. The process of differentiation of vegetal plate cell types might involve lineage segregation and cellular interactions of contiguous founder cell populations such as the gut cells. For the migratory cell types like the pigment cells or basal cells, differentiation might be lineage independent and, like vertebrate neural crest cells, rely on information from the positions to which the cells move.

This research was supported by NSF grant DCB-8911829 to R.A.C., by NIH grant HD-25138 to S.E.F., and by NIH grant HD-05753 to E.H.D.

\section{References}

AMEMIYA, S. (1989). Electron microscopic studies on primary mesenchyme cell ingression and gastrulation in relation to vegetal pole cell behavior in sea urchin embryos. Expl Cell Res. $183,453-462$.

Amemiya, S., Akasaki, K. and Terayama, H. (1982). Scanning electron microscopy of gastrulation in a sea urchin (Anthocidaris crassispina). J. Embryol. exp. Morph. 67, 27-35.

BuRkE, R. D. (1978). The structure of the nervous system of the pluteus larva of Strongylocentrotus purpuratus. Cell Tissue Res. 191, 233-247.

Burke, R. D. (1980). Morphogenesis of the digestive tract of the pluteus larva of Strongylocentrotus purpuratus: shaping and bending. Int. J. Invertebr. Reprod. 2, 13-21.

Burke, R. D. And Alvarez, C. M. (1988). Development of the esophageal muscles in embryos of the sea urchin Strongylocentrotus purpuratus. Cell Tissue Res. 252, 411-417.

Burke, R. D. ANd ChlA, F.-S. (1980). Morphogenesis of the digestive tract of the pluteus larva of Strongylocentrorus purpuratus: sphincter formation. Int. J. Invertebr. Reprod. 2, $1-12$

Cameron, R. A. and Davidson, E. H. (1991). Cell type specification during sea urchin development. TIG 7, 212-218

Cameron, R. A., Fraser, S. E., Brtten, R. J. and Davidson, E. H. (1989). The oral-aboral axis of the sea urchin embryo is specified by first cleavage. Development 106, 641-647. 
Cameron, R. A., Fraser, S. E., Britten, R. J. and Davidson, E. H. (1990). Segregation of oral from aboral ectoderm precursors is completed at fifth cleavage in the embryogenesis of Strongylocentrotus purpuratus. Devl Biol. 137, 77-85.

Cameron, R. A., Hough-Evans, B. R., Britten, R. J. and Davidson, E. H. (1987). Lineage and fate of each blastomere of the eight-cell sea urchun embryo. Genes Dev. 1, 75-84.

CzIHAK, G. (1971). Echinoids. In Experimental Embryology of Marine and Freshwater Invertebrates (ed. G. Reverberi), pp. 363-506. Amsterdam, London: North-Holland Publ. Co.

Davidson, E. H. (1986). Gene Activity in Early Development, Thurd edition. Orlando, Florida: Academic Press.

Davidson, E. H. (1989). Lineage-specific gene expression and the regulative capacities of the sea urchin embryo: a proposed mechanism. Development 105, 421-445.

Ettensohn, C. A. (1984). Primary invagination of the vegetal plate during sea urchin gastrulation. Am. Zool. 24, 571-588.

Ettensohn, C. A. (1985). Gastrulation in the sea urchin embryo is accompanied by the rearrangement of invaginating epithelial cells. Devl Biol. 112, 383-390.

Gibson, A. W. and Burke, R. D. (1985). The origin of pigment cells in embryos of the sea urchin Strongylocentrotus purpuratus. Devl Biol. 107, 414-419.

Gibson, A. W. AND Burke, R. D. (1987). Migratory and invasive behavior of pigment cells in normal and animalized sea urchin embryos. Expl Cell Res. 173, 546-557.

Gustafson, T. AND Wolpert, L. (1963a). Studies on the cellular basis of morphogenesis in the sea urchin embryo. Formation of the coelom, the mouth, and the primary pore-canal. Expl Cell Res. 29, 561-582.

Gustafson, T. and Wolpert, L. (1963b). The cellular basis of morphogenesis and sea urchin development. Int. Rev. Cytol. 15, 139-214.

Hardin, J. and Cheng, L. Y. (1986). The mechanisms and mechanics of archenteron elongation during sea urchin gastrulation. Devl Btol. 115, 490-501.

Hörstadius, S. (1939). The mechanisms of sea urchin development, studied by operative methods. Biol. Rev. Cambridge Phil. Soc. 14, 132-179.

Hour, M. S. AND Hinegardner, R. T. (1980). The formation and early differentiation of sea urchin gonads. Biol Bull. mar. biol. Lab., Woods Hole 159, 280-294.

Houk, M. S. and Hinfgardner, R. T. (1981). Cytoplasmic inclusions specific to the sea urchin germ line. Dev/ Biol. 86, 94-99.

Ishimoda-TakaGi, T., Chino, I. and Sato, H. (1984). Evidence for the involvement of muscle tropomyosin in contractile elements of the coelom-esophagus complex in sea urchin embryos. Devl Biol. 105, 365-376.

MacBride, E. (1903). The development of Echinus esculentus, together with some notes on the development of $E$ muliaris and E. acutus. Phil. Trans. Roy. Soc. London 195B, 285-330.

McMahon, A. P., Flytzanis, C. N., Hough-Evans, B. R. Katula, K. S., Britten, R. J. and Davidson, E. H. (1985). Introduction of cloned DNA into sea urchin egg cytoplasm: Replication and persistence durng embryogenesis. Devl Biol. 108, 420-430.

Morrill, J. B. ANd SANTos, L. (1985). A scanning electron microscopical overview of cellular and extracellular patterns during blastulation and gastrulation in the sea urchin, Lytechtmus variegatus. In The Cellular and Molecular Biology of Invertebrate Development (ed. R. Sawyer and R. Showman), pp. 3-34. Columbia, SC: University of South Carolina Press.

Nislow, C. ANd Morrill, J, B. (1988). Regional cell division during sea urchin gastrulation contributes to archenteron formation and is correlated with the establishment of larval symmetry. Dev. Growth Differ. 30, 483-499.

OKAZAKI, K. (1975). Normal development to metamorphosis. In The Sea Urchin Embryo. Biochemistry and Morphogenesis (ed. G. Czihak), pp. 177-222. Berlin: Springer-Verlag.

Pearse, J. S. and Cameron, R. A. (1991). Echinoids. In Reproduction in Marine Intertebrates. Boxwood Press, Pacific Grove, CA.

Pehrson, J. and Cohen, L. (1986). The fate of the small micromeres in sea urchin development. Devl Biol. 113, 522-526.

RYBERG, E. AND LuNDGREN, B. (1977). Extra-ectodermal strands in the ciliated bands of the echinopluteus. Dev. Growth Diff. 19 , $299-308$.

Snedecor, G. W. and Cochran, W. G. (1979). Statustical Methods. Ames, IA: Iowa State University Press.

TANAKA, S. AND DAN, K. (1990). Study of the lineage and cell cycle of small micromeres in embryos of the sea urchin, Hemicentrotus pulcherrimus. Dev. Growth Differ. 32, 145-156.

Young, R. S. (1958). Development of pigment in the larva of the sea urchin, Lytechinus variegatus. Biol. Bull. mar. biol. Lab., Woods Hole 114, 394-403.

(Accepted 28 August 1991) 